\title{
Energy injection in short GRBs and the role of magnetars
}

\author{
A. Rowlinson \\ Astronomical Institute "Anton Pannekoek", University of Amsterdam, Postbus 94249, 1090 GE \\ Amsterdam, The Netherlands \\ E-mail: b.a.rowlinson@uva.nl \\ P. T. O'Brien* \\ Department of Physics \& Astronomy, University of Leicester, University Road, Leicester, LE1 \\ 7RH, United Kingdom \\ E-mail: paul.obrien@leicester.ac.uk
}

\begin{abstract}
A significant fraction of the Long Gamma-ray Bursts (GRBs) in the Swift sample show a plateau phase which may be due to ongoing energy injection. We find many Short GRBs detected by the Swift satellite show similar behavior. The remnant of NS-NS mergers may not collapse immediately to a BH (or even collapse at all) forming instead a magnetar. This model predicts that there would be a plateau phase in the X-ray lightcurve followed by a shallow decay phase, if it is a stable magnetar, or a steep decay if the magnetar collapses to a BH. By fitting this model to all of the Short GRB BAT-XRT lightcurves, we find that a significant fraction may show evidence of energy injection by a magnetar. This model can be tested using the next generations of gravitational wave observatories.
\end{abstract}

Gamma-Ray Bursts 2012 Conference-GRB2012,

May 07-11, 2012

Munich, Germany

${ }^{*}$ Speaker. 


\section{Introduction}

The Swift satellite [10] has revolutionised the study of early X-ray emission in gamma-ray bursts (GRBs). Swift observations led to the discovery of features such as flares and plateaus in many long GRB (LGRB) lightcurves, showing evidence of prolonged energy injection from the central engine [21, 23, 31]. Also the rapid slewing capabilities of Swift enabled the detection of the faint and rapidly decaying short GRB (SGRB) X-ray afterglows which has led to a number of candidate host galaxy identifications [11,13]. The afterglow properites and identified host galaxies of SGRBs are consistent with the popular progenitor theory: the merger of two neutron stars or a neutron star and black hole forming a black hole [15, 8, 20]. This progenitor theory requires confirmation via the coincident detection of gravitational waves and a SGRB.

With the increasing number of SGRB X-ray afterglows, it has become apparent that SGRBs also have features caused by energy injection in their lightcurves. However, this is problematic for SGRB progenitor theories as accretion is expected to end within a few seconds and there is only a small amount of material ejected into eccentric orbits which may accrete at late times [25, 18]. An alternative model is that the merger of two neutron stars does not form a black hole, instead coalescing to form a highly magnetised, rapidly rotating pulsar (magnetar) which has enough rotational energy to prevent immediate gravitational collapse to a black hole [29, 7, 5]. As the magnetar spins down, its rotational energy is transfered to graviational waves and electromagnetic radiation. During this spin down process the magnetar may reach a critical point, at which it can no longer support its mass via rapid rotation, resulting in collapse to a black hole. This critical point is highly dependent on the equation of state of a neutron star. Following the recent discovery of a $\sim 2 \mathrm{M}_{\odot}$ neutron star [6], it has been shown that many neutron star mergers may result in a delayed collapse to a black hole or may not collapse at all [24].

Assuming constant radiative efficiency, the electromagnetic energy injection from the magnetar produces a plateau in the X-ray lightcurve [30]. If the magnetar is stable the plateau will be followed by a shallow decay phase as it spins down, however if it is unstable there will be a steep decay phase in the lightcurve when the magnetar collapses to form a black hole. This plateau and steep decay phase has been observed in several LGRB and SGRB lightcurves [28, 17, 26].

Here we consider all Swift SGRBs detected by the Burst Alert Telescope (BAT) with $T_{90} \leq 2$ s, until March 2012, which were promptly slewed to and observed by the Swift X-ray Telescope (XRT). We identify those with a plateau phase in their lightcurves suggesting ongoing energy injection from the central engine. For 28 SGRBs with sufficient data, we fit the magnetar model directly to the lightcurves. This work is described in detail in Rowlinson et al. (2012, submitted to MNRAS).

\section{Evidence of energy injection in SGRB lightcurves}

The combined BAT-XRT lightcurves for all the Swift SGRBs have been fitted using a broken power-law utilising the method described in [9]. We find $~ 50 \%$ of SGRBs have a shallow decay, or plateau, phase showing evidence of energy injection. The SGRBs which required 2 or more breaks in their afterglows are plotted in Figure 1. In Figure 1, we compare the lightcurve break times 

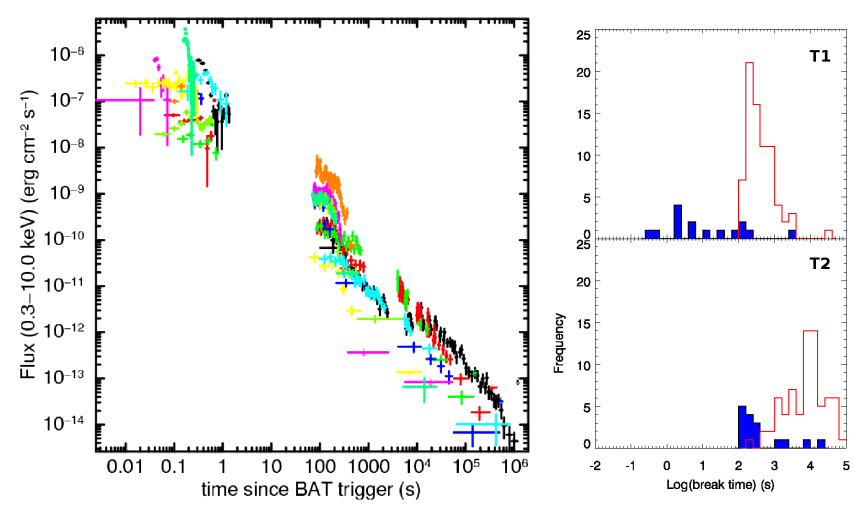

Figure 1: Left: These GRBs have 2 or more breaks in their lightcurve: Black - 051221A, red - 060313, green - 061201, dark blue - 070724, light blue - 090426, magenta - 090515, yellow - 100625A, orange - 100702A, light green - 101219A, cyan - 120305A. Right: Histograms showing the break times for the SGRB lightcurves with a plateau phase. $\mathrm{T}_{1}$ is the break from the steep decay phase to the plateau phase while $\mathrm{T}_{2}$ marks the end of the plateau. The blue filled histograms correspond to the SGRB sample used in this Paper and overplotted in red are the LGRB values determined by [9].

to those for LGRBs showing that the SGRB breaks, before and after plateau phases, are occuring orders of magnitude earlier than those for LGRBs.

\section{Fitting the magnetar model}

The model fitted in this work is as described in [30] and has previously been applied by [28, 17, 26]. This model is consistent to the late-time residual spin-down regime described in [19]. We fit the equations below directly to the observed data which has been extrapolated to the restframe using a k-correction [2] (when no redshift was available, we used $\mathrm{z} \sim 0.7$ ). The data were fit with an additional underlying power-law component whose decay rate is governed by the curvature effect [14]. In some cases, we allowed the power-law component to vary. These equations apply to the electromagnetic dominated spin down regime, as the gravitational wave dominated regime would be extremely rapid and produce a negligble electromagnetic signal. We have assumed that the emission is $100 \%$ efficient and isotropic.

$$
\begin{array}{r}
B_{p, 15}^{2}=4.2025 I_{45}^{2} R_{6}^{-6} L_{0,49}^{-1} T_{e m, 3}^{-2} \\
P_{0,-3}^{2}=2.05 I_{45} L_{0,49}^{-1} T_{e m, 3}^{-1} \\
L_{e m, 49}(T)=L_{0,49}\left(1+\frac{T}{10^{-3} T_{e m, 3}}\right)^{-2}
\end{array}
$$

where $T_{e m, 3}$ is the plateau duration in $10^{3} \mathrm{~s}, L_{0,49}$ is the plateau luminosity in $10^{49} \mathrm{erg} \mathrm{s}^{-1}$, $L_{e m, 49}(T)$ is the time-dependent luminosity in $10^{49} \mathrm{erg} \mathrm{s}^{-1}, I_{45}$ is the moment of inertia in units of $10^{45} \mathrm{~g} \mathrm{~cm}^{2}, B_{p, 15}$ is the magnetic field strength at the poles in units of $10^{15} G, R_{6}$ is the radius of the neutron star in $10^{6} \mathrm{~cm}$ and $P_{0,-3}$ is the initial period of the compact object in milliseconds. The equations of vacuum dipole spin-down given above neglect the enhanced angular momentum losses due to neutrino-driven mass loss, which are important at early times after the magnetar forms [19]. 

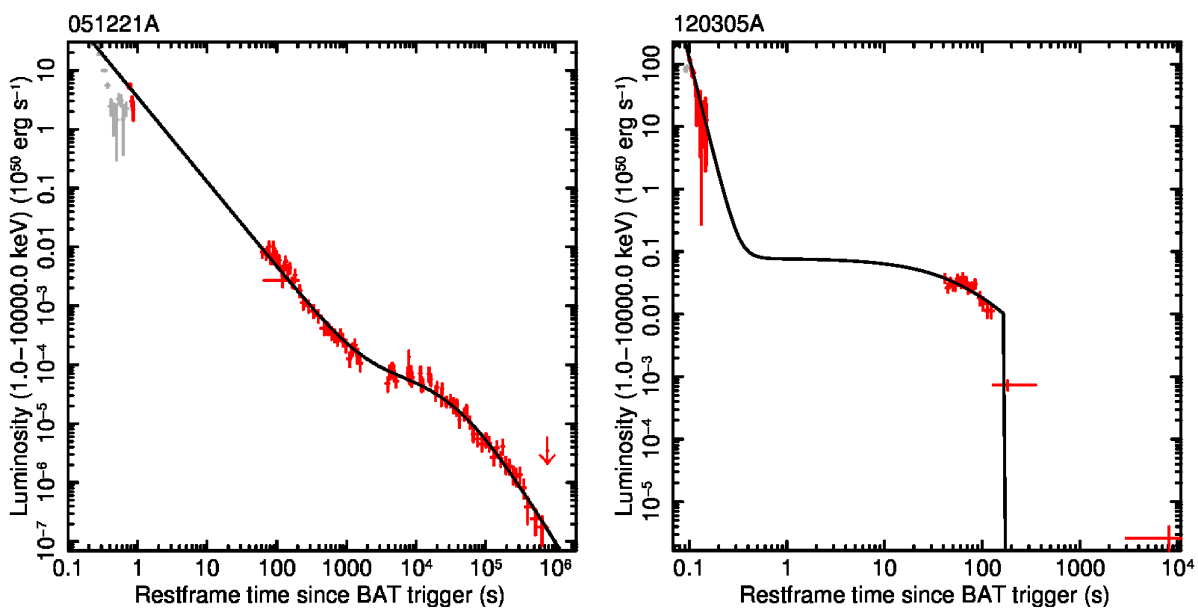

Figure 2: Example fits for two of the SGRBs in this sample. GRB 051221A (left) is best fit by a stable magnetar and GRB 120305A (right) is best fit by a magnetar which collapses to a black hole at $\sim 200 \mathrm{~s}$. The grey data points are excluded from the fit.

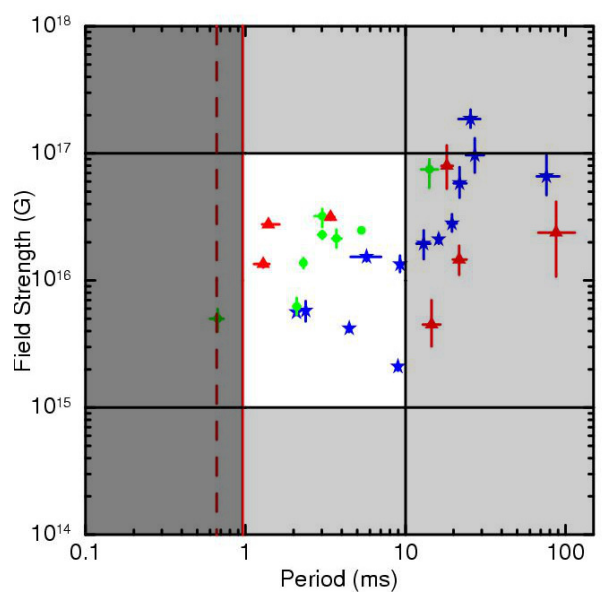

Figure 3: A graph showing the magnetic field and spin period of the magnetar fits produced. The solid (dashed) red line represent the spin break up period for a $1.4 \mathrm{M}_{\odot}\left(2.1 \mathrm{M}_{\odot}\right)$ neutron star [16] and the unshaded region shows the expected region for an unstable pulsar, as defined in [17] and [26]. The initial rotation period needs to be $\leq 10 \mathrm{~ms}$ [29] and the lower limit for the magnetic field is $\geq 10^{15} \mathrm{G}$ [27]. Blue stars $=$ good fit to the magnetar model with a stable magnetar, Green circles $=$ good fit to the model with an unstable magnetar which collapses to form a BH, and Red triangles = poor fit to the model.

Nevertheless, these expressions reasonably approximate the spin-down of very highly magnetized neutron stars of most relevance in this paper. Isotropic emission is also a reasonable assumption for relatively powerful magnetar winds, since (unlike following the collapse of a massive star) the magnetar outflow cannot be confined efficiently by the relatively small quantity of surrounding material expected following a NS merger [3]. 


\section{Results}

28 SGRBs had sufficient data for fitting to the magnetar model and $\sim 75 \%$ had a good fit to the model. For the SGRBs which had a poor fit this may simply be due to insufficient data or flares. Example fits to the magnetar model are shown in Figure 2 for GRB 051221A, consistent with the formation of a stable magnetar, and GRB 120305A which has a steep decay phase associated with the magnetar collapsing to form a black hole at $\sim 200 \mathrm{~s}$.

The derived magnetic field strengths and spin periods are plotted in Figure 3. All candidates have spin periods in excess of the shortest allowed spin periods. The lines in the figure represent the predicted maximum/minimum values for the magnetic fields and an estimated maximum spin period. Almost all of the magnetar candidates lie within the expected magnetic field strengths, although some have relatively low spin periods.

\section{Gravitational wave signals}

This model may be testable via the detection of gravitational waves from all three phases of this model: insprial to form a magnetar [1], the spin down of the magnetar [4] and, for the unstable magnetar candidates, the collapse to form a black hole [22]. Although the inspiral phase is going to have the brightest associated gravitational wave signal, Advanced LIGO may be able to detect all three phases for sources within $100 \mathrm{Mpc}$ (445 Mpc for the inspiral phase only) although the expected rates are very low for the simultaneous detection of gravitational waves and electromagnetic radiation. Using the predicted sensitivity for the Einstein Telescope [12], the detection distance limits are increased to within $1300 \mathrm{Mpc}$ (5900 Mpc for the inspiral phase only) and this will provide a significant increase to the expected rates.

The detection of multiple gravitational wave signals with associated features in the gamma-ray and X-ray lightcurves will provide an ideal test of the magnetar model.

\section{References}

[1] Abadie J., et al., TOPICAL REVIEW: Predictions for the rates of compact binary coalescences observable by ground-based gravitational-wave detectors, CQGra, 27 (2010) 173001

[2] Bloom J. S., Frail D. A., Sari R., The Prompt Energy Release of Gamma-Ray Bursts using a Cosmological k-Correction, AJ, 121 (2001) 2879

[3] Bucciantini N., Metzger B. D., Thompson T. A., Quataert E., Short gamma-ray bursts with extended emission from magnetar birth: jet formation and collimation, MNRAS, $\mathbf{4 1 9}$ (2011) 1537

[4] Corsi A., Mészáros P., Gamma-ray Burst Afterglow Plateaus and Gravitational Waves: Multi-messenger Signature of a Millisecond Magnetar?, ApJ, 702 (2009) 1171

[5] Dai Z. G., Lu T., Gamma-ray burst afterglows and evolution of postburst fireballs with energy injection from strongly magnetic millisecond pulsars, A\&A, 333 (1998) L87

[6] Demorest P. B., Pennucci T., Ransom S. M., Roberts M. S. E., Hessels J. W. T., A two-solar-mass neutron star measured using Shapiro delay, Nature, 467 (2010) 1081

[7] Duncan R. C., Thompson C., Formation of very strongly magnetized neutron stars - Implications for gamma-ray bursts, ApJ, 392 (1992) L9 
[8] Eichler, D., Livio, M., Piran, T., Schramm, D. N., Nucleosynthesis, neutrino bursts and gamma-rays from coalescing neutron stars, Nature, 340 (1989) 126

[9] Evans P. A., et al., Methods and results of an automatic analysis of a complete sample of Swift-XRT observations of GRBs, MNRAS, 397 (2009) 1177

[10] Gehrels N., et al., The Swift Gamma-Ray Burst Mission, ApJ, 611 (2004) 1005

[11] Gehrels N., et al., A short Îs-ray burst apparently associated with an elliptical galaxy at redshift $z=$ 0.225, Nature, 437 (2005) 851

[12] Hild S., et al., Sensitivity studies for third-generation gravitational wave observatories, CQGra, 28 (2011) 094013

[13] Hjorth J., et al., GRB 050509B: Constraints on Short Gamma-Ray Burst Models, ApJ, 630 (2005) L117

[14] Kumar P., Panaitescu A., Afterglow Emission from Naked Gamma-Ray Bursts, ApJ, 541 (2000) L51

[15] Lattimer, J. M., Schramm, D. N., The tidal disruption of neutron stars by black holes in close binaries, ApJ, 210 (1976) 549

[16] Lattimer J. M., Prakash M., The Physics of Neutron Stars, Sci, 304 (2004) 536

[17] Lyons N., O’Brien P. T., Zhang B., Willingale R., Troja E., Starling R. L. C., Can X-ray emission powered by a spinning-down magnetar explain some gamma-ray burst light-curve features?, MNRAS, 402 (2010) 705

[18] Metzger B. D., Piro A. L., Quataert E., Time-dependent models of accretion discs formed from compact object mergers, MNRAS, 390 (2008) 781

[19] Metzger B. D., Giannios D., Thompson T. A., Bucciantini N., Quataert E., The protomagnetar model for gamma-ray bursts, MNRAS, 413 (2011) 2031

[20] Narayan, R., Paczynski, B., Piran, T., Gamma-ray bursts as the death throes of massive binary stars, ApJ, 395 (1992) L83

[21] Nousek J. A., et al., Evidence for a Canonical Gamma-Ray Burst Afterglow Light Curve in the Swift XRT Data, ApJ, 642 (2006) 389

[22] Novak J., Spherical neutron star collapse toward a black hole in a tensor-scalar theory of gravity, PhRvD, 57 (1998) 4789

[23] O’Brien, P. T., et al., The Early X-Ray Emission from GRBs, ApJ, 647 (2006) 1213 [astro-ph/0601125]

[24] Ozel F., Psaltis D., Ransom S., Demorest P., Alford M., The Massive Pulsar PSR J1614-2230: Linking Quantum Chromodynamics, Gamma-ray Bursts, and Gravitational Wave Astronomy, ApJ, 724 (2010) L199

[25] Rosswog S., Fallback accretion in the aftermath of a compact binary merger, MNRAS, 376 (2007) L48

[26] Rowlinson, A., et al. The unusual X-ray emission of the short Swift GRB 090515: evidence for the formation of a magnetar?, MNRAS, 409 (2010) 531

[27] Thompson T. A., Assessing Millisecond Proto-Magnetars as GRB Central Engines, RMxAC, 27 (2007) 80 
[28] Troja E., et al., Swift Observations of GRB 070110: An Extraordinary X-Ray Afterglow Powered by the Central Engine, ApJ, 665 (2007) 599

[29] Usov V. V., Millisecond pulsars with extremely strong magnetic fields as a cosmological source of gamma-ray bursts, Nature, 357 (1992) 472

[30] Zhang B., Mészáros P., Gamma-Ray Burst Afterglow with Continuous Energy Injection: Signature of a Highly Magnetized Millisecond Pulsar, ApJ, 552 (2001) L35

[31] Zhang, B., Fan, Y. Z., Dyks, J., Kobayashi, S., Mészáros, P., Burrows, D. N., Nousek, J. A., \& Gehrels, N., Physical Processes Shaping Gamma-Ray Burst X-Ray Afterglow Light Curves: Theoretical Implications from the Swift X-Ray Telescope Observations, ApJ, 642 (2006) 354 